\title{
Research on Injection Rate Allocation Fault Diagnosis in Integrated Separate Layer Water Injection Technology
}

\author{
Deli Jia ${ }^{12^{*}}$, Xiaohan $\mathrm{Pei}^{1}$, He Liu ${ }^{1}$, Fuchao Sun ${ }^{1}$ and Mingxin $\mathrm{Zhao}^{3}$ \\ 1. China Research institute of oil exploration and development (RIPED), Petro \\ China, Beijing 100083, China \\ 2. School of Automation, Harbin University of Science \&Technology, Harbin 150080, \\ China \\ 3. Production Engineering Research Institute, Daqing Oilfield Company Ltd, Daqing \\ 163453, China \\ E-mail: jiadeli@hrbust.edu.cn
}

\begin{abstract}
Integrated separate layer water injection technology is an effective approach to reduce the workload of injection oilfield. The primary equipment which consists of flowmeter, motor and control value is placed underground for a long time. Injection rate allocation fault diagnosis is a problem with character of multiformity. The fault symptoms and reasons are random, and it is hard to differentiate them. Weighted fuzzy $C$-means clustering algorithm combining symptoms signals and fuzzy mathematics approach is used on the technology. The algorithm is based on fuzzy C-means clustering algorithm, and increase weight to each fault sample to reduce the impact of samples on fault classifications. Simulation and analysis illustrates that the algorithm is able to reflect the real structure of the sample data. And it improves the accuracy of fault classification.
\end{abstract}

Keywords: separated layer water injection, integration, fuzzy algorithm, clustering algorithm, fault

\section{Introduction}

Research and technological innovation have been made to meet the demands of different stages, solve the contradiction between layers and achieve efficient water injection. The ommingled water injection expanded towards separate layer water injection [1]. The running and pulling of pipes have developed to nozzles adjustment and direct-readout. The data can be collected by multi-parameter, which was collected by single-parameter [2]. And it can be storage electrically, which was recorded on cards. The data can be direct-readout [3]. With the increasing of injection wells and water injection layers, the workload of construction operations is increased year by year. Therefore, free throw integrated separate layer injection was developed, that combined the detection with resolution and was put down the hole [4-5]. The tools always break down under high temperature and pressure that would reduce injection rate and water flooding. The artificial fault diagnosis used for regular inspection is essential to popularize and apply the process.

With the development of the fuzzy theory [6], artificial neural networks [7], genetic algorithm [8], gray clustering and expert systems [9] and other intelligence theory, fault diagnosis system becomes more intelligent, various intelligent algorithms play their own advantages and features in fault diagnosis. In integrated separate layer water injection 
technology, injection rate allocation process will cause fault for many reasons, which is characterized by diverse, and there are fuzziness and randomness between fault symptoms and fault reasons, so it's difficult to establish fault diagnosis model. Fuzzy theory is one way that can accurate description of the uncertainty relations, the signal of fault symptoms combined with fuzzy mathematics can effectively improve the accuracy of fault diagnosis. Therefore, this paper proposes a C-means clustering algorithm based on weighted fuzzy for injection rate allocation fault diagnosis in integrated separate layer water injection technology.

\section{Downhole Injection Rate Allocation Process Analysis}

The underground water distributor is used for injection allocation in integrated separate layer water injection technology. The water distributor structure is shown in figure 1, which consists of mechanical body, cylinder pipe cable, monitoring circuit, pressure gauge, flowmeter, control valve and motor. And the technology process is that injected water flow along coiled oilpipes to the main channel of water distributor, through control valve into the single injected channel, then through the flowmeter into the water injection. The pressure gauge is used to detect the injection pressure of main channel. The flowmeter detects monolayer injection amount. The motor controls opening of control valve to adjust monolayer injection amount, and realize the monolayer closed-loop flow control.

The flowmeter, motor and control valve should maintain stable operating in the process of injection rate allocation. The underground electric controlled parts always break down under high temperature and pressure that brings latent danger to the flow allocation. And they need periodic measuring. The reasons of fault can provide the basis for the follow-up maintenance work. Then the fault detection can only depend on limited electrical information, which obtained ground communication by cylinder pipe cable. So artificial intelligence method must be used to raise the accuracy, reduce error and improve efficiency.

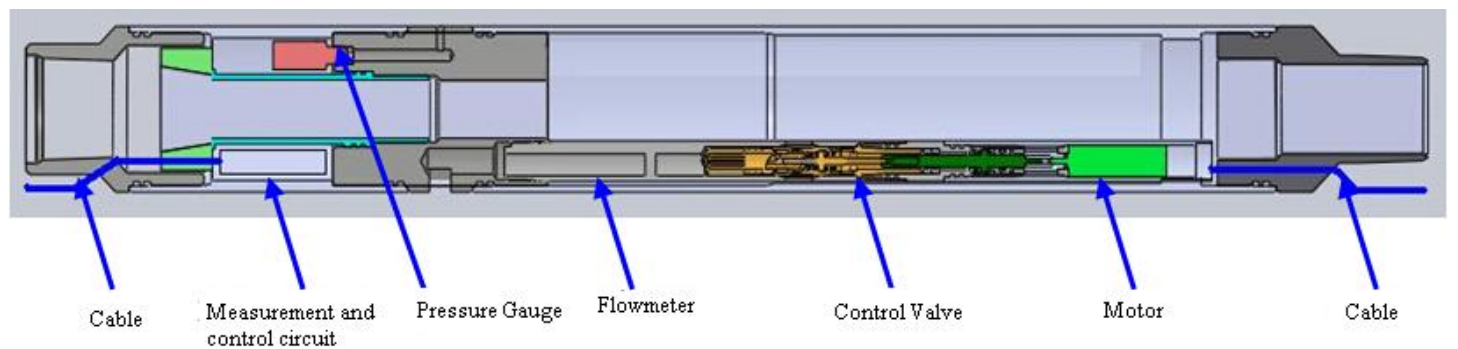

Figure 1. Downhole Dynamometer Process Principles

\section{WFCM Fault Diagnosis System}

The traditional classification methods (such as the classification of the three ratio method and classification of the specialist experience, etc., ) can't get the real structure of fault data samples. The traditional classification methods always first to determine the failure mode, then according the certain rules or experience to distribute the fault sample into different classification of the fault, but doing this is not a scientific analysis method of sample data. In the transformer fault diagnosis, there are extremely complex randomness and fuzziness between fault reason and fault symptom.

The clustering is a process that according to the similarity to distinguish and classify in things. The clustering is a kind of unsupervised classification, and that divide the class is 
unknown. In 1969, fuzzy clustering was first formulated and studied by Ruspini [10]. Because the fuzzy clustering shows the intermediary of generic sample, it can objectively reflect the real world, becoming the mainstream of the cluster analysis.

The algorithm of Fuzzy C-Means (Fuzzy, c-Means, FCM) [11] is the most perfect, the most widely used algorithm, which based on fuzzy clustering algorithm theory of the objective function. The FCM algorithm is from the hard C-Means clustering (HCM) algorithm evolved. FCM algorithm has been shown to have good convergence [12]

Hypothesis $X=\left\{x_{1}, x_{2}, \cdots, x_{n}\right\}$ is limited numbers of clustering analysis, Among them $X=\left\{x_{1}, x_{2}, \cdots, x_{n}\right\}$ said the $s$ eigenvalues in the $j$ samples, The cluster analysis for the given data set $X$ is the $X$ is divided into subsets $c$, means that there are $c$ subset $X_{1}, X_{2}, \cdots, X_{c}$, set the clustering center of $c$ subset for $V=\left\{v_{1}, v_{2}, \cdots, v_{c}\right\}, c$ is the integer in accordance with $2 \leq c \leq n$.Using the membership function $u_{i j}=u_{x_{i}}\left(x_{j}\right)$ to show the subordinate relationship between Sample $x_{j}$ and subset $X_{i}(1 \leq i \leq c)$. The interval $\{0,1\}$ of membership function expands to the interval $[0,1]$ by Ruspini using fuzzy set theory (hard cdividing). Based on flexible dividing fuzzy cluster analysis algorithm, c-dividing space is below:

$$
M_{f c}=\left\{U \in R^{c u} \mid u_{i j} \in[0,1], \forall i, j ; \sum_{i=1}^{c} u_{i j}=1, \forall j ; 0<\sum_{j=1}^{n} u_{i j}<n, \forall i\right\}
$$

Simultaneously introduced weighted index $m$, FCM algorithm objective function is:

$$
\left\{\begin{array}{c}
J(U, V)=\sum_{i=1}^{c} \sum_{j=1}^{n} u_{i j}^{m} d_{i j}^{2} \\
\text { s.t. } \quad U \in M_{f c}
\end{array}\right.
$$

In the formula, $m$ is weighted index (Smoothing parameter); $d_{i j}\left(d_{i j}=\left\|x_{j}-v_{i}\right\|\right)$ is the distance between sample $x_{j}$ and the center of $i$ class.

$U=\left[u_{i j}\right]$ is Membership matrix, $u_{i j}$ is sample $x_{j}$ belonging to various categories of membership matrix. Fuzzy clustering criterion is determined appropriate fuzzy partition matrix $U=\left\{u_{i j}\right\}$ and cluster centers $v_{i}$ to reach the minimum of the objective function $J(U, V)$. Since among each column in the fuzzy partition matrix $U$ are independent, so we can get:

$$
\min \{J(U, V)\}=\min \left\{\sum_{i=1}^{c} \sum_{j=1}^{n} u_{i j}^{m} d_{i j}^{2}\right\}=\sum_{j=1}^{n} \min \left\{\sum_{i=1}^{c} u_{i j}^{m} d_{i j}^{2}\right\}
$$

The constraint condition of above extremum is $\sum_{i=1}^{c} u_{i j}=1$. It can use Lagrange multiplier method to construct objective function to solve minimum of $J(U, V)$. Literature [13-14] showed that constrains above cannot reflect of samples typicalness, and were inapplicable in the case that samples of data sets differ greatly in quantity. In fault diagnosis, there are different eigenvalues and electrical parameters in different fault patterns. For there is big difference between the point of cluster center calculated by FCM and fact, it is hard to be classified correctly(not be discussed here). Therefore, weighted fuzzy C-means clustering algorithm is used for fault diagnosis to improve the accuracy of cluster center. There are different classified patterns in different samples of various parts. In order to obtain correct 
classification, the position of cluster center can be adjusted. The weight enhances performance of algorithm. The option of weight is a key to improve algorithm [15].

For each sample $x_{i}$, the density function is:

$$
\begin{aligned}
z_{i} & =\sum_{j=1, j \neq i}^{n} \frac{1}{D_{i j}^{1 / a}}, 1 \leq i \leq n \\
D_{i j} & =\left\|x_{i}-x_{j}\right\|, 1 \leq i, j \leq n
\end{aligned}
$$

In the expression, $D_{i j}$ is euclidean distance between sample point $x_{i}$ and $x_{j}, a$ is a parameter, $a \geq 1$.

If there are more sample around $x_{i}, z_{i}$ is more bigger, or not, $z_{i}$ is smaller. $e$ is density range limited value, $\min \left(D_{i j}\right)<e<\max \left(D_{i j}\right)$. After $z_{i}$ is normalized, the weight matrix is below:

$$
w_{i}=\frac{z_{i}}{\sum_{j=1}^{n} z_{j}}, 1 \leq i \leq n
$$

Bring the weighting matrix to FCM algorithm, as the formula 3, it can get the objective function improved:

$$
\min \{J(U, V, W)\}=\sum_{i=1}^{c} \sum_{j=1}^{n} w_{j} u_{i j}^{m} d_{i j}^{2}
$$

It is obtained:

$$
\left\{\begin{array}{c}
u_{i j} \in[0,1], 1 \leq i \leq c, 1 \leq j \leq n \\
\sum_{i=1}^{c} u_{i j}=1,1 \leq j \leq n \\
0<\sum_{j=1}^{n} u_{i j}<n, 1 \leq i \leq c
\end{array}\right.
$$

To get appropriate $U=\left\{u_{i j}\right\}$ and $v_{i}$ to obtain the minimum value $J(U, V, W)$. In a similar way, using lagrange multiplier method to construct a new objective function for solving the minimum of the formula 8 .

$$
\begin{aligned}
\hat{J}(U, V, W) & =J(U, V, W)+\sum_{j=1}^{n} \lambda_{j}\left(\sum_{i=1}^{c} u_{i j}-1\right) \\
& =\sum_{i=1}^{c} \sum_{j=1}^{n} w_{j} u_{i j}^{m} d_{i j}^{2}+\sum_{j=1}^{n} \lambda_{j}\left(\sum_{i=1}^{c} u_{i j}-1\right)
\end{aligned}
$$

In the formula $9, \lambda \quad\left(\lambda=\left\{\lambda_{1}, \lambda_{2}, \cdots, \lambda_{n}\right\}\right)$ were Lagrange multipliers of constraints.

Derivative operation for all input parameters in the formula 9, the process to obtain the minimum value $u_{i j}$ and $v_{i}$ of the objective function, specific methods are as follows.

From $\partial \hat{J} / \partial v_{i}=0$, we can get:

$$
v_{i}=\sum_{j=1}^{n} w_{j} u_{i j}^{m} x_{j} / \sum_{j=1}^{n} w_{j} u_{i j}^{m}, 1 \leq i \leq c
$$

From $\partial \hat{J} / \partial u_{i j}=0$, we can get: 


$$
u_{i j}=\left(\frac{\lambda}{m v_{i} d_{i j}^{2}}\right)^{\frac{1}{m-1}}, 1 \leq i \leq c, 1 \leq j \leq n
$$

Due to the constraint condition $\sum_{i=1}^{c} u_{i j}=1$, summation of the $\mathrm{i}$ in the formula 11:

$$
\lambda^{\frac{1}{m-1}}=\frac{1}{\sum_{k=1}^{c}\left(\frac{1}{m w_{j} d_{k j}^{2}}\right)^{\frac{1}{m}}}
$$

Taking the formula 11 into formula 12, can get:

$$
u_{i j}=\frac{1}{\sum_{k=1}^{c}\left(\frac{d_{i j}}{d_{k j}}\right) \frac{2}{m-1}}, 1 \leq i \leq c, 1 \leq j \leq n
$$

All above, accomplish the algorithm about specific weighted fuzzy $c$ means clustering.

Step1: Inputing clustering dataset $X=\left\{x_{1}, x_{2}, \cdots, x_{n}\right\}$, giving cluster category $c$, defining weighting exponent $m$, setting an arbitrary small iteration error $\varepsilon>0$, max iteration number $T_{\max }$ of the algorithm.

Step2: According to the formula 4-6, calculation weighting matrix $w_{i}$.

Step3: Initialization fuzzy membership matrix to satisfy the constraints.

Step4: Calculation clustering center $v_{i}^{t}(i=1,2, \cdots c)$.

Step5: Objective function calculation, determined the convergence of the algorithm in the iterative process.

Step6: According to the formula 13 and $v_{i}^{t}$, updating $U^{(t)}$ of $U^{(t+1)}$.

Step7:If $\left\|U^{(t+1)}-U^{(t)}\right\|<\varepsilon$ then stop iterative, or set $t=t+1$ and return to step4.

Getting the the optimal fuzzy partition matrix $U^{*}$ and cluster centers $v_{i}{ }^{*}$, We can identify the attribution of each sample by Center recently principle or maximum membership principle.

\section{Simulation and Application of Engineering}

\subsection{Select and Normalized Eigenvalues}

Injection rate allocation of long time operation in integrated separate layer water injection technology will show common faults: Not centered and unbalanced of the motor rotor causes the drive current is too high $I_{\text {overcurent; }}$ As the motor seepage, motor insulation level is not enough $\mathrm{V}$; when the flowmeter probe is not complete failure, the linearity deviation will migration $\mathrm{F}$ (Abbreviation flowmeter migration); ceramic faucets of clogged will damage and the faucets will failure D due to sundries clogging (shows flow leakage exists in the degree of opening theory).

Selected characteristic signals are: drive voltage, drive current, useful power of the motor, the signal of the flowmeter output (frequency), and feedback of the motor encoder. Due to the different motor drive power and clogging model number, the drive current and leakage eigenvalues have big difference. So normalizing the samples, each value is limited $[0,1]$, To deal with the following: 


$$
x_{i j}^{\prime}=\frac{x_{i j}}{\sum_{k=1}^{5} x_{i k}}, 1 \leq i \leq n, 1 \leq j, k \leq 5
$$

In the formula, $x_{i j}$ is original sample data (Eigenvalues offset), $x_{i j}^{\prime}$ is the value of the

normalized original data, and $\sum_{j=1}^{5} x_{i j}^{\prime}=1 . i$ is the sample number, $j$ is attribute number, respectively denote eigenvalues according to the above 5 fault.

\subsection{Fault Diagnosis Examples and Analysis}

Injection rate allocation fault in integrated separate layer water injection technology is diagnosed by WFCM algorithm and FCM algorithm using Matlab. There are four faults metioned in the technology. This paper collected 100 fault samples. $v_{1}, v_{2}, v_{3}, v_{4}$ indicate the cluster centers of $I_{\text {overcurent }}, \mathrm{V}, \mathrm{F}, \mathrm{D}$ respectively. The parameters of WFCM algorithm are below: $m$ equals 2 , iterative error $\varepsilon$ equals $10-3$, the optimum maximum number of iterations $T_{\max }$ equals 100 , cluster categories $\alpha$ equals 4 (It was proved that the cluster accuracy is towards atability $90 \%$, when $\alpha$ is greater than 4), The same things is done with FCM algorithm. The results are shown in Table 1 and Table 2.

FCM algorithm and WFCM algorithm converges to the global minimum point with 34 and 25 iterations respectively. The contrast experimentsusing using these two algorithms are shown in Table 1. The accuracy using WFCM algorithm was obviously higher than using FCM algorithm. For the error rate in WFCM is lower than in FCM shown in Table 2, the fault can be classified better by using WFCM than FCM. For the error square using WFCM is lower than using FCM, the point of cluster center is more reasonable by using WFCM than FCM.

The practical exprements' results are shown Table 3, that the algorithm made in this paper was applied to injection rate allocation fault diagnosis. Compared with traditional three-ratio method and FCM, WFCM can get satisfied results for 5 examples.

Table 1. Contrast the Diagnosis Results of FCM and WFCM Methods

\begin{tabular}{c|c|c|c|c|c}
\hline \multirow{2}{*}{ Fault type } & \multirow{2}{*}{ Numbers } & \multicolumn{2}{|c|}{ The arithmetic of FCM } & \multicolumn{2}{c}{ The arithmetic of WFCM } \\
\cline { 3 - 6 } & & Correct numbers & Accuracy\% & Correct numbers & Accuracy\% \\
\hline $\mathrm{I}_{\text {overcurrent }}$ & 14 & 10 & 71.43 & 12 & 85.71 \\
\hline $\mathrm{V}$ & 16 & 12 & 75 & 14 & 87.5 \\
\hline $\mathrm{F}$ & 24 & 15 & 62.5 & 20 & 83.33 \\
\hline $\mathrm{D}$ & 46 & 30 & 65.22 & 40 & 86.96 \\
\hline Total & 100 & 67 & 67 & 86 & 86 \\
\hline
\end{tabular}

Table 2. Effect of the FCM and WFCM Diagnosis Methods

\begin{tabular}{c|c|c|c|c}
\hline Algorithm & Iteration times & Points wrong number & Misclassification rate\% & Error sum of squares \\
\hline FCM & 34 & 33 & 33 & 1.584554 \\
\hline WFCM & 25 & 14 & 14 & 0.021473 \\
\hline
\end{tabular}


Table 3. Fault Diagnosis Example

\begin{tabular}{c|c|c|c|c}
\hline Sample number & Three ratios diagnosis & FCM diagnosis & WFCM diagnosis & True fault \\
\hline 1 & $\mathrm{D}$ & Ivvercurrent & Ivverurrent & Iovercurrent \\
\hline 2 & $\mathrm{~V}$ & $\mathrm{~V}$ & $\mathrm{~V}$ & $\mathrm{~V}$ \\
\hline 3 & $\mathrm{~F}$ & $\mathrm{~F}$ & $\mathrm{~F}$ & $\mathrm{~F}$ \\
\hline 4 & Ioverurrent & $\mathrm{I}_{\text {overcurrent }}$ & $\mathrm{D}$ & $\mathrm{D}$ \\
\hline 5 & $\mathrm{D}$ & $\mathrm{D}$ & $\mathrm{D}$ & $\mathrm{D}$ \\
\hline
\end{tabular}

\section{Conclusion}

Integrated separate layer water injection technology functions as a technical support for improving the level of water-driven oil in oilfield. It is important to implement the process that the latent failures in injection rate allocation process can be diagnosed accurately and timely. The accuracy of the traditional diagnostic method depends on the accumulation of experience, but diagnostic accuracy of the results is not high. In addition, the faulty would have been only diagnosed by limited electrical information. So a new method is mentioned in this paper that combined fault symptoms with fuzzy mathematics, and added weights to each sample. And it is used for fault diagnosis in the injection rate allocation. Compared the results of simulation and examples it is known that compared with the traditional three-ratio diagnosis and FCM diagnosis, WFCM algorithm makes the cluster center closer to the actual position. The algorithm can effectively improve the accuracy of fault diagnosis, and can be used in practical engineering.

\section{Acknowledgements}

This work is supported by National High Technology Research and Development Program (863 Program), China (2012AA061300).

This work is also supported by Harbin special funds for technological innovation talent development research. (2014RFQXJ165)

\section{References}

[1]. G. Zhenbao and W. Xiufen, "Review and outlook of separate layer water injection technology in Daqing Oilfield", Special Oil \& Gas Reservoirs, vol. 13, (2006), pp. 4-9.

[2]. L. Yongsheng "Intelligent linkage adjusting system for separate injection well", Petroleum Instruments, vol. 21, (2007), pp. 62-63.

[3]. L. He, P. Xiaohan, L. Kai, S. Fuchao, Z. Lichen and Y. Qinghai. "Current status and trend of separated layer water flooding in China", Petroleum Exploration and Development, vol. 40, (2013), pp. 733-737.

[4]. J. Deli, X. Shengnan, W. Yao and C. Cui, "Control strategy of flow on single layer of injection well", 2012 International Conference on Measurement, Information and Control, MIC 2012, vol. 2, (2012), pp. 765-768.

[5]. D. Jia, F. Wang, J. Xu and S. Xu, "Variable-universe adaptive fuzzy control of layered water injection technique, Electric Machines and Control”, vol. 16, (2002), pp. 66-70.

[6]. S. Caixin, G. Junfeng, L. Ruijing, etc., "Study on Fault Diagnose Method of Transformer Dga with Fuzzy Model Hiberarchy Classification", Chinese Society for Electrical Engineering", vol. 21, (2001), pp. 37-41.

[7]. Z. Jianhua and H. Minqiang, "Application of Auto-Structural Neural Network in Diagnosing Transformer Faults", China Electrotechnical Society, vol. 19, (2004), pp. 77-81.

[8]. Y C. Huang, "Condition Assessment of Power Transformers Using Genetic based Neural Networks", IEEE Proceedings-Science, Measurement and Technology, vol. 150, (2003), pp. 19-24.

[9]. L. Jian, S. Caixin and C. Weigen. "Study on Fault Diagnosis of Insulation of Oil-immersed Transformer Based on Grey Cluster Theory", China Electrotechnical Society, vol. 17, (2002), pp. 80-83.

[10]. W. Zhongdong, G. Xinbo, X. Weixin, etc., "Parallel fuzzy clustering algorithm based on kernel method", Journal of the Xi'an University of Electronic Science and Technology, vol. 31, (2004), pp. 533-537.

[11]. G. Xinbo, "Fuzzy Clustering Analysis and Applications", Xi'an: Xi'an University of Electronic Science and Technology Press, (2004). 
International Journal of Control and Automation

Vol. 7, No. 12 (2014)

[12]. J C. Bezdek, "A convergence theorem for the fuzzy ISODATA clustering algorithms", IEEE Trans on Pattern Anal Mach Intel, vol. 2, (1980), pp. 1-8.

[13]. R. Krishnapurarm and J. M. Kell, “A possilistic approach to clustering”, IEEE Trans. FS, vol. 1, (1993), pp. 98-110

[14]. J C. Bezdek, "Pattern recognition with fuzzy objective function algorithms", Plenum Press, New York, (1981).

[15]. F. Jiulun, "Studies on New Fuzzy Clustering Algorithms and Clustering Validity Problems", Xi'an, Xi'an University of Electronic Science and Technology Doctoral Dissertation, (1981). 